\title{
A new method for the estimation of the onset of the lateralized readiness potential (LRP)
}

\author{
P. SCHWARZENAU, M. FALKENSTEIN, J. HOORMANN, and J. HOHNSBEIN \\ University of Dortmund, Dortmund, Germany
}

\begin{abstract}
The aim of this work was to develop a new method for the estimation of the onset of the lateralized readiness potential (LRP). In contrast to the known methods that use only restricted data segments for estimation, the proposed segmented regression (SR) method employs the LRP trace from the stimulus onset to the LRP peak. Comparison of the SR with two other methods, done with both simulated and real data, shows that the SR method yields a relatively unbiased absolute onset time that is not affected by different LRP gradients before and after the onset. Moreover, the SR estimator proved to be quite sensitive to subtle onset differences across conditions.
\end{abstract}

The lateralized readiness potential (LRP) is a transient asymmetry of activity over the two lateral motor areas, which is observed shortly before an overt response or an attempt to respond (Coles \& Gratton, 1986; de Jong, Wierda, Mulder, \& Mulder, 1988; Smid, Mulder, \& Mulder, 1987). It is usually measured as the difference between the averaged EEG activity recorded from the centrolateral electrodes over the motor areas $\left(\mathrm{C}^{\prime}{ }^{\prime}\right.$ and $\left.\mathrm{C}^{\prime}\right)$. The LRP can be observed with maximum amplitude in choice reaction tasks that involve the two hands. According to the formula of de Jong et al. (1988), positive values of the LRP denote preparation of the wrong hand, whereas negative values denote preparation of the correct hand. In recent years, the LRP has gained considerable attention, because it is recognized as an objective correlate of covert reaction tendencies (cf. also the contribution of Eimer, 1998). Recently it has been shown that the LRP is not tightly coupled with the overt response, but that the overt response can be delayed in relation to the LRP (Falkenstein, Hohnsbein, \& Hoormann, 1994). Falkenstein et al. (1994), among others, have also shown that the latency of the peak of the LRP and its onset can vary differentially. When they manipulated the complexity of the task (two vs. four response alternatives), a delay in reaction time (RT) of about $100 \mathrm{msec}$ resulted. In the ERPs, the LRP onset was delayed by the same amount $(63 \mathrm{msec})$ as was the latency of the late P300 subcomponent (P-CR, which is linked to the central decision process, or response identification) $(65 \mathrm{msec})$. In contrast, the peak of the LRP was delayed by $85 \mathrm{msec}$. Falkenstein et al. (1994) thus concluded that the LRP onset was more closely time-related to central decision processes, whereas the LRP peak was more closely related to the actual response - that is, to central motor processes. Hence the LRP onset yields information that is different from the information delivered by the LRP peak latency.

Correspondence concerning this article should be sent to $\mathrm{M}$. Falkenstein, Institut für Arbeitsphysiologie an der Universität Dortmund, Ardeystr. 67, D-44139 Dortmund, Germany (e-mail: falkenstein@ arb-phys.uni-dortmund.de).
Several methods have been proposed for the estimation of the LRP onset. Smid et al. (1987) estimated the LRP onset by calculating a series of Wilcoxon rank sum tests (one Wilcoxon score for each sample) for the amplitude differences between left- and right-hand response trials. The onset of the LRP was defined (in a series of $t$ tests) as the sample point at which the Wilcoxon statistic deviates significantly from zero. This method is time consuming, since data evaluation on the single trial level is necessary. In all other methods, the LRP onset is calculated from waveforms averaged across a certain number of single trials. A problem with such LRP onset estimation from averages is that comparisons with RTs are biased. The average RT is computed from the onsets of the overt response, and all responses have the same weight. In contrast, the onset of average waveforms such as the LRP will be determined mainly on the basis of trials in which the LRP occurs relatively early (Meyer, Osman, Irwin, \& Yantis, 1988). Since the LRP varies roughly with RT, the onset in the averages will be more determined by the fast responses, whereas the slow responses will have much less influence. This asymmetry generates a number of discrepancies between the two measures. One way to reduce this problem is to compute separate LRPs for trials in which the RT is relatively constant, as we describe below.

Recently two relatively simple methods for the onset estimation of averaged LRPs have been described by Osman and Moore (1993) and Smulders, Kenemans, and Kok (1996). The first method (that of Osman and Moore) is based on an idea similar to that behind Smid et al.'s (1987) method-namely, to define the LRP onset as a deviation from a baseline. In this method (henceforth called the baseline deviation, or $\mathrm{BD}$, method), the baseline of the LRP trace - that is, the period $50 \mathrm{msec}$ before to $100 \mathrm{msec}$ after stimulus onset-is determined first. Then its mean $\left(y_{\mathrm{b}}\right)$ and standard deviation $(s)$ across samples are calculated. The LRP onset latency $t_{0}$ is defined as that point at which the LRP values $y(t)$ become larger (more negative) and stay larger than $2 s$ of the baseline mean $y_{\mathrm{b}}$ for a certain period of time (e.g., $100 \mathrm{msec}$ ): 


$$
y\left(t_{0}+t\right)<y_{\mathrm{b}}-2 s, \text { for } t=1, \ldots, 100 .
$$

This method does not make use of the entire database, but only of the first part of the LRP trace, the baseline.

The second method (Smulders et al., 1996) defines the LRP onset as that point, $t_{0}$, at which a certain proportion (preferably 50\%) of the peak amplitude of the LRP is reached. This method (henceforth called the peak proportion, or PP, method) again does not use the entire database (similar to the BD method) but only the later part of the LRP trace. It should be mentioned that Smulders et al. (1996) also consider other variants of the $50 \%$ methodfor example, a $25 \%$ or $12.5 \%$ threshold. However, since the $50 \%$ level highlights the problem of taking the LRP peak as anchor for estimating the onset latency, we will focus our arguments on this value.

Very recently, Miller, Patterson, and Ulrich (1998) have proposed a jackknife method to measure and test statistically LRP onset differences between conditions. This method measures the LRP onset latency as the first time point at which the grand average (wave shape averaged across all subjects) exceeds a predefined criterion. By subtracting these values for two conditions, one obtains the LRP onset latency difference, $D$, between the conditions. To be able to test such differences statistically, one estimates the standard error by jackknifing (see Miller, et al. 1998). The advantage of the jackknife method is that the database for LRP onset estimation contains only averages based on $N$ (grand averages) or $N-1$ subjects, which are relatively noise-free wave shapes. As for the absolute values of LRP onset latencies, this method also uses the BD principle noted above. Hence the problems of the BD method, as discussed below, should also apply to the jackknife method.

\section{New Method: Segmented Regression (SR)}

When one scans grand averages of LRP traces in the literature, it becomes evident that the LRP time course between stimulus onset and LRP peak usually consists of two roughly linear segments with different gradients. Hence the onset of the LRP can be straightforwardly defined as the bend of the trace - the beginning of the segment that eventually reaches the peak. This definition is in accordance with how the onset is basically estimated when clean LRP data are examined by eye.

An LRP trace can thus be approximated by a segmented regression model with two different straight lines (Seber $\&$ Wild, 1989), where the LRP onset $t_{0}$ is determined as the point of intersection of the two straight lines, according to the following formulae:

$$
\begin{aligned}
& y(t)=f(t)+e, \\
& f(t)=\mathrm{b}_{11}+\mathrm{b}_{12}, \text { for } t_{\mathrm{a}}<t<t_{0},
\end{aligned}
$$

and

$$
f(t)=\mathrm{b}_{21}+\mathrm{b}_{22} t, \text { for } t_{0}<t<t_{\mathrm{e}},
$$

where $y(t)$ is the LRP trace, $f(t)$ the two-line model, $e$ the noise, $t_{\mathrm{a}}$ the beginning, and $t_{\mathrm{e}}$ the end of the approximated LRP trace, or the LRP peak latency. In our application of the model, we set $t_{\mathrm{a}}$ to $50 \mathrm{msec}$ after stimulus onset. Without any further constraints, the two lines were adjusted to yield a minimum deviation from the real data (minimum of the sum of squared deviations). The advantage of this method is that the entire LRP trace from the baseline to the peak latency is used, so that probably a better estimator of the onset latency is obtained. The SR method is based on average ERPs (as are the BD and PP methods) and not on single trials (as is the method of Smid et al., 1987). Hence, it is less time consuming than the latter method.

\section{Comparison of the SR and the Other Methods}

The already mentioned problem with the BD and PP methods is that the LRP onset estimation is based on restricted parts of the LRP time course. Hence, systematic upward or downward trends before and after the LRP onset are not considered, and these may influence the onset estimation.

Early trends before LRP onset are found often in the literature. They occur on fast trials, for example, in which subjects prepare (prematurely) for one hand without having finished the stimulus-response mapping (e.g., Ulrich, Moore, \& Osman, 1993). In cases of preinformation, such trends are also clearly seen (e.g., Ulrich et al., 1993). Furthermore, early trends opposite to the final LRP direction can be found in the literature (e.g., Leuthold \& Sommer, 1993), as well as in our own data. As an example, an individual LRP trace from a choice RT task is shown in Figure 1 . This trace reveals an upward trend, followed by a sharp and steady downward trend. With the BD method, the LRP onset latency is estimated to be $350 \mathrm{msec}$, which is clearly too large. The same applies to the PP method: when the LRP peak amplitude is measured relative to technical zero, the estimated latency is about $330 \mathrm{msec}$ (the point where $50 \%$ of the peak amplitude of $6 \mu \mathrm{V}$ relative to zero is reached). When the peak amplitude is measured relative to the mean baseline value $(-2.7 \mu \mathrm{V})$, the PP-estimated onset is even much later (about $360 \mathrm{msec}$ ). In this case, the onset estimation is influenced not only by the early trend, but also by a possible nonzero baseline. The SR method yields an LRP onset at $286 \mathrm{msec}$, where the two regression lines (Figure 1, dashed lines) intersect. Hence, the onset estimated with the SR method coincides well with the beginning of the monotonous downward flank of the LRP, whereas the other two methods estimate the onset to be much later.

An overestimation may not necessarily be a problem, if it is constant across conditions. However, this is clearly not the case with the BD and PP methods. Figure 2 illustrates schematically how downward (upper panel) and upward (lower panel) early trends differentially influence the onset of the LRP as estimated by the BD method $\left(t_{00}\right)$ : For a downward trend, the onset is estimated to occur about $70 \mathrm{msec}$ before the bend $\left(t_{\mathrm{c}}\right)$; for an upward trend, it is estimated to be at about $70 \mathrm{msec}$ after the bend. Hence it is evident that the direction of the trend heavily influences the LRP onset estimated with the BD method. In certain cases with opposite early trend and relatively small LRP amplitude (e.g., the dotted line after the LRP peak), the BD method is likely to find no LRP onset at all, because 


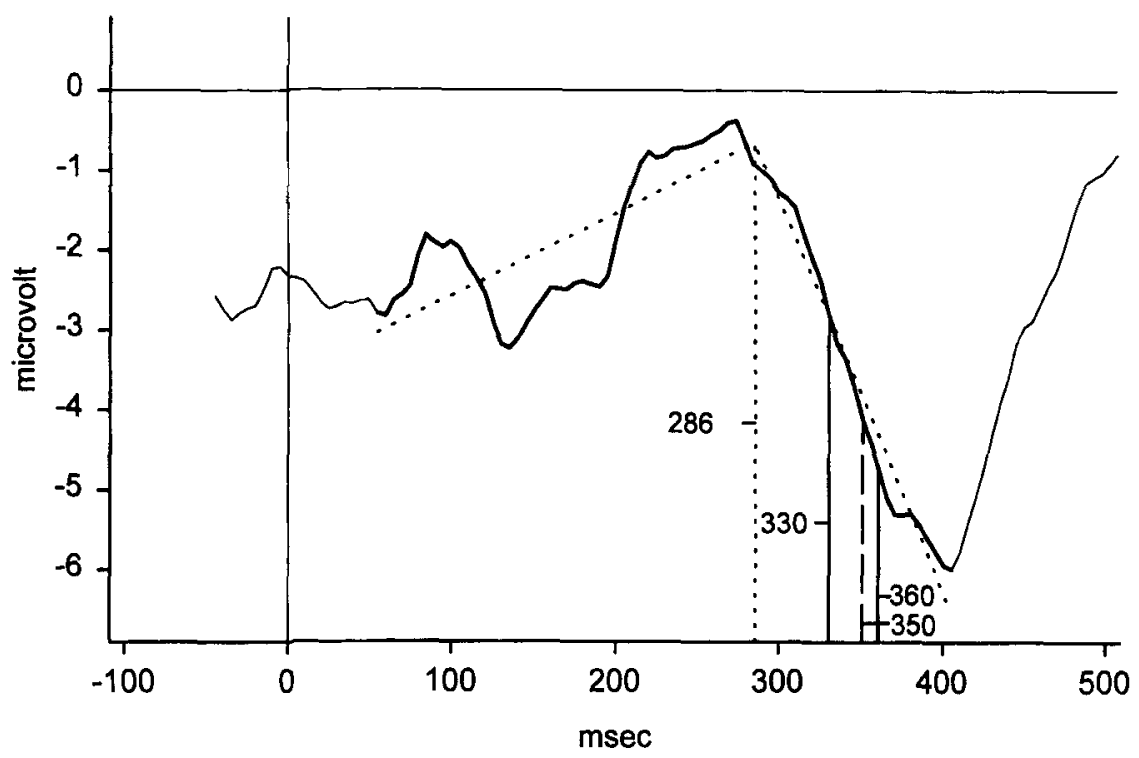

Figure 1. Individual LRP trace in a two-alternative choice reaction experiment. There is a negative baseline around stimulus onset $(0)$, followed by an upward trend, followed by a sharp downward slope. The dotted line illustrates the estimated regression lines within the segmented regression (SR) model. The thick part of the LRP trace is taken for SR estimation. The vertical line yields the estimated onset values with the SR method (dotted line, $286 \mathrm{msec}$ ), as well as with the known methods BD (dashed line, $350 \mathrm{msec}$ ) and PP (straight lines, $330 \mathrm{msec}, 360 \mathrm{msec}$ ). (The 360 -msec value is obtained when the LRP peak amplitude is measured relative to the mean baseline $I-50$ to +50 msec around stimulus onset; thin tracel).

the LRP does not stay more than $100 \mathrm{msec}$ below the $2 s$ level (second criterion of Formula 1).

Similar differences result if the onset is estimated with the PP method $\left(t_{0 \mathrm{~s}}\right)$ : If we assume that the LRP peak amplitude (related to the average baseline, which is, for simplicity, assumed to be equivalent to zero) is constant across conditions, the estimated onset values are 370 (upward trend) and 305 (downward trend).

Late trends of the LRP after the bend also affect the estimation with the known methods, particularly the PP method. First, the estimation with the PP method depends on the peak amplitude of the LRP. If the amplitude varies across conditions, the estimated onset latency varies accordingly, although the bend latency may be constant. Second, even if one assumes that the LRP amplitude is invariant across conditions and there is no early trend in the LRP, a severe problem remains if there are different trends in the second segment of the LRP between conditions.

Figure 3 illustrates schematically how different slopes of the LRP after the bend can influence the onset estimation with the PP method of Smulders et al. $\left(1996 ; t_{0 \mathrm{~s}}\right)$. In the upper panel, a constant bend latency $\left(t_{\mathrm{c}}\right)$ with different slopes of the LRP after the bend is assumed. This leads to a latency difference of $70 \mathrm{msec}$, estimated with the PP method. In contrast, the example in the lower panel shows that even in the case of different bend latencies across conditions, different slopes after the onset can yield the same onset estimation with the PP method $\left(t_{0 \mathrm{~s}}\right)$. Hence, the LRP onset estimation with the PP method is influenced not only by the amplitude of the baseline (if the peak amplitude is related to the baseline), but also by the LRP peak latency, which may vary differently from the LRP onset latency, as mentioned above.

\section{Comparison of the \\ Three Methods Using Real Data}

The following example with real data demonstrates the existence of such slope differences across conditions and how they influence the LRP onset estimation and the results in an analysis of variance (ANOVA).

\section{Experimental Design and Data Processing}

Visual and auditory letter stimuli ( $\mathrm{F}$ or $\mathrm{J}$ ) were presented with a random interstimulus interval of 950 and $2,050 \mathrm{msec}$; stimulus modality and letter name were randomized within a block. Since letter modality was unpredictable, the subjects had to divide their attention between both modalities (divided attention paradigm; Hohnsbein, Falkenstein, Hoormann, \& Blanke, 1991). Ten subjects performed two-alternative choice reactions with the right and the left index finger to each letter ( $\mathrm{J}$ and $\mathrm{F}$, respectively). The subjects had to respond within a time limit of $500 \mathrm{msec}$. Slower responses were signaled by a feedback tone, which had to be avoided. The EEG was measured at four midline electrodes $(\mathrm{Fz}, \mathrm{Cz}, \mathrm{Pz}, \mathrm{Oz})$ and at $\mathrm{C}^{\prime}$ and $\mathrm{C}^{\prime}$. The ERPs of the correct trials were averaged with the stimulus onset as trigger. The LRP was computed from the ERP difference waves between $\mathrm{C}^{\prime}$ and $\mathrm{C}^{\prime}$ ', using the 

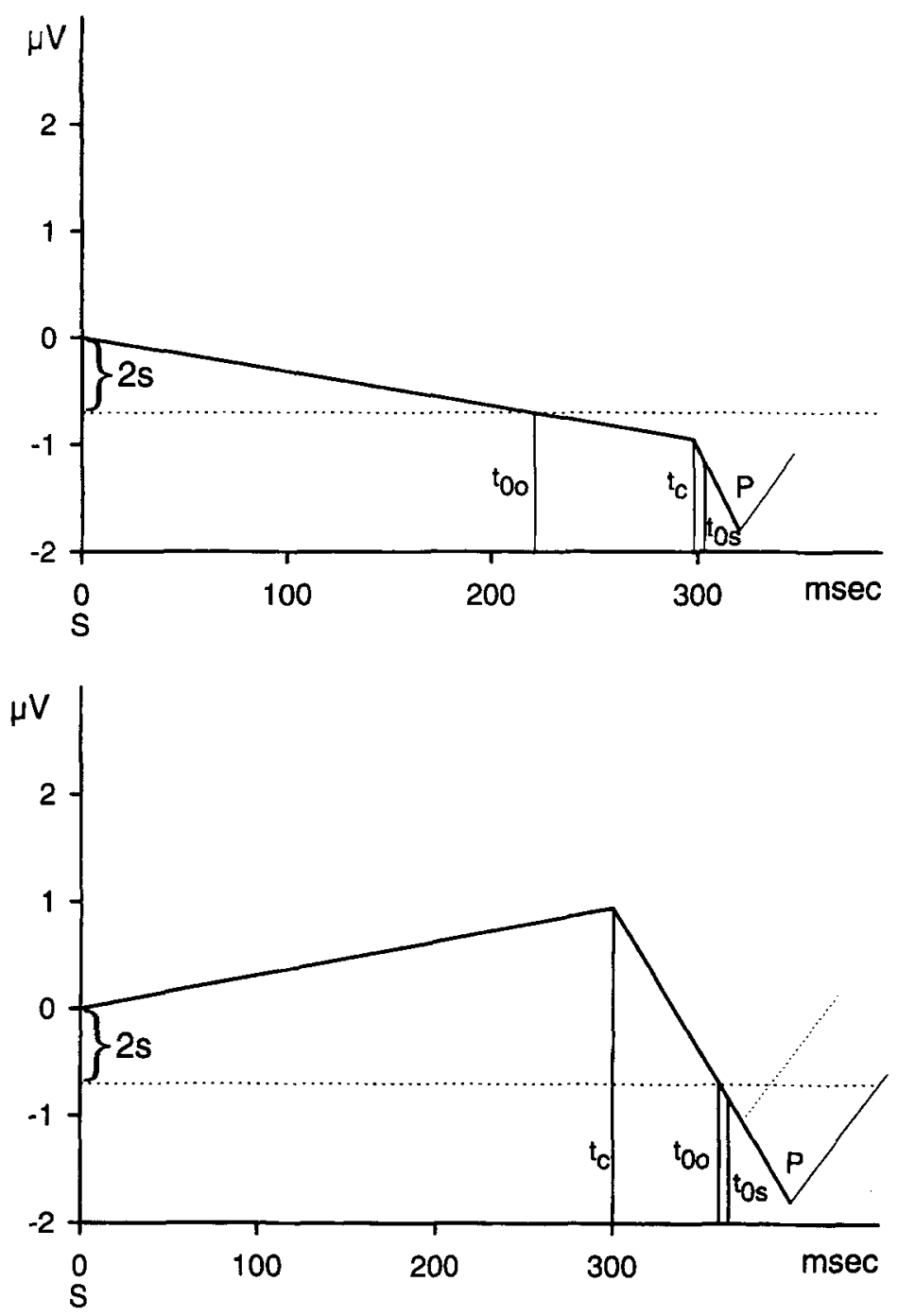

Figure 2. Schematic illustrations of how downward (upper panel) and upward (lower panel) early trends before the LRP onset influence its estimation with the BD method of Osman and Moore (1993) $\left(t_{00}\right)$ as well as with the PP method of Smulders et al. (1996) $\left(t_{0 \mathrm{~s}}\right)$, while the bend $\left(t_{\mathrm{c}}\right)$ has a constant latency of $300 \mathrm{msec}$. The dashed line in the lower panel shows how an LRP onset can be missed when the 100-msec criterion of the BD method is not met. P, LRP peak.

double subtraction method (de Jong et al., 1988; Smid et al., 1987):

$$
\mathrm{LRP}=\left(\mathrm{C} 3^{\prime}-\mathrm{C} 4^{\prime}\right) \text { right hand }-\left(\mathrm{C3}^{\prime}-\mathrm{C} 4^{\prime}\right) \text { left hand. }
$$

In an attempt to decrease the bias toward fast responses to the LRP onset (see the introduction) and to reduce the time jitter and hence "smearing" of the LRP slope, the RT variance was reduced by two techniques: First, we excluded trials with very fast and slow RTs (below $100 \mathrm{msec}$ and above $500 \mathrm{msec}$ ). Second, for the remaining trials, we computed for each subject LRP subaverages for slow (RT above median) and fast (RT below median) responses, which yielded the two post hoc conditions fast and slow. On the average, the RTs were about $100 \mathrm{msec}$ longer for slow than for fast responses. The subjects spontaneously showed large performance differences (as revealed in their error rates); therefore, they were divided into two groups: poor ( 5 subjects with an error rate above the median; mean error rate, $21 \%$ ), and good ( 5 subjects with an error rate below the median; mean error rate, $6 \%$ ). As reported earlier (Falkenstein, Hohnsbein, \& Hoormann, 1996), the RTs were shorter for the poor subjects after auditory but not after visual stimuli (a significant group $\times$ modality interaction). Given the differences in performance, differences in the LRP onset were assumed between the groups. A mixed ANOVA design with the following factors resulted: performance group (good, poor), modality (visual, auditory), and response speed (slow, fast). The dependent 

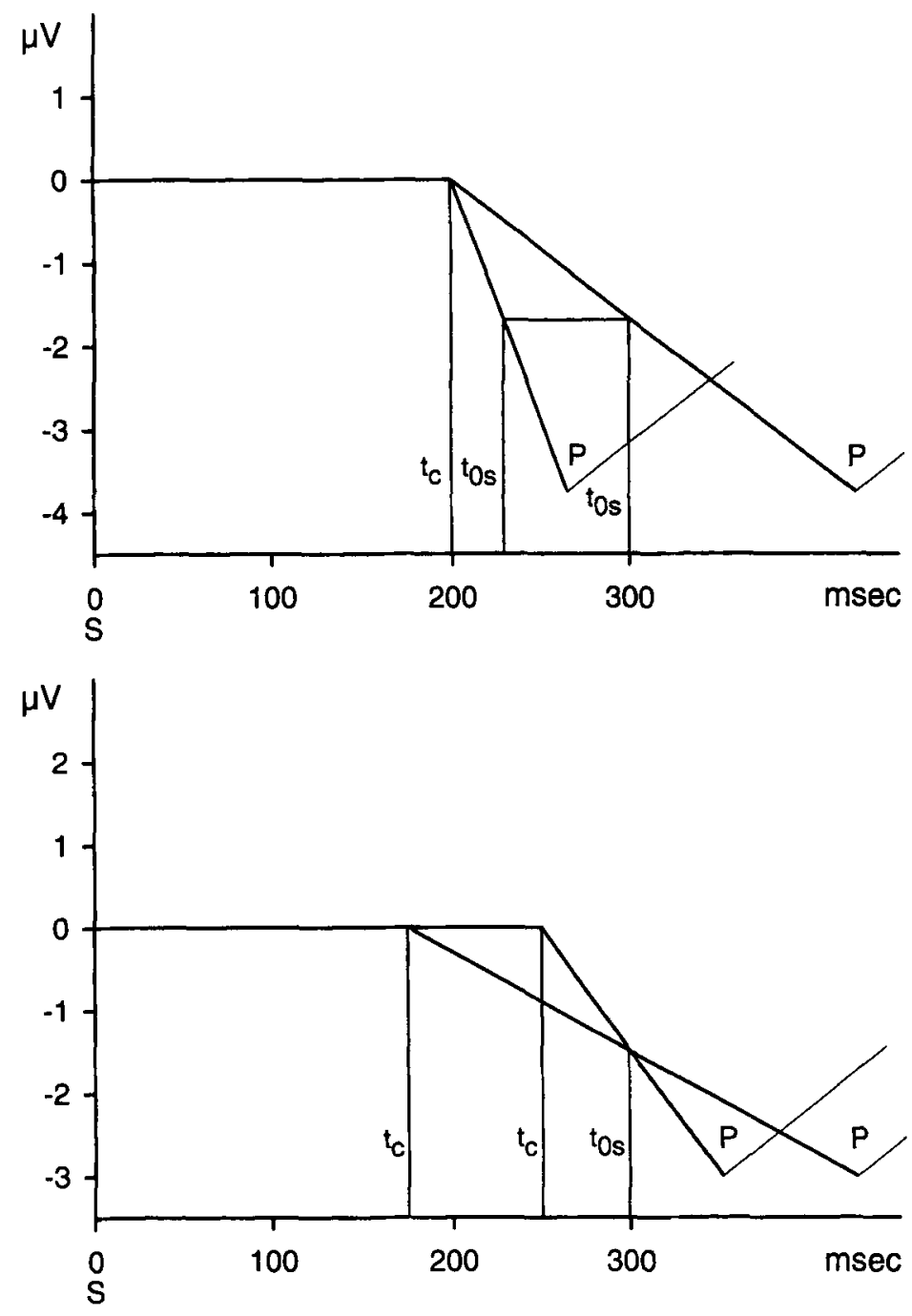

Figure 3. Schematic illustrations of how different slopes of the LRP after the bend can influence the onset estimation with the PP method of Smulders et al. (1996) $\left(t_{0 s}\right)$. In the upper panel a stable bend $\left(t_{c}\right)$ with different slopes after the break is assumed, which leads to different latencies estimated with the PP method. The lower panel shows that even in the case of different bend latencies across conditions different slopes after the onset can yield the same onset estimation $\left(t_{0 s}\right)$. P, LRP peak.

variable was the LRP onset, estimated with the three methods (BD, PP, RS).

\section{Results}

Figure 4 shows the LRPs for both groups, subdivided for slow and fast responses (pooled across modalities for a better overview). The two-segment character of all LRP curves from stimulus onset to the peak is obvious, exhibiting a fairly distinct bend between the first and the second segment. The estimated onset values are marked with vertical bars for the three methods. The SR-estimated onsets are in good concordance with the bends for all conditions. The onsets estimated with the other methods are fairly late.
As expected, the LRPs of slow (vs. fast) responses are delayed by about $100 \mathrm{msec}$, which mirrors the RT difference between the bins. For slow responses, the LRP onset shows no group difference, whereas for fast responses, the LRP begins earlier for poor subjects, revealed in the bends and with the SR method. Hence the data suggest a significant group $\times$ speed interaction (no difference for slow, difference for fast responses) for the LRP onset latency.

When the LRPs of the fast responses were plotted for both groups and modalities (Figure 5), it turned out that the earlier onset for poor subjects occurred mainly after auditory stimuli. This early LRP onset after auditory stimuli mirrors the behavioral data (shorter RTs after auditory stimuli 


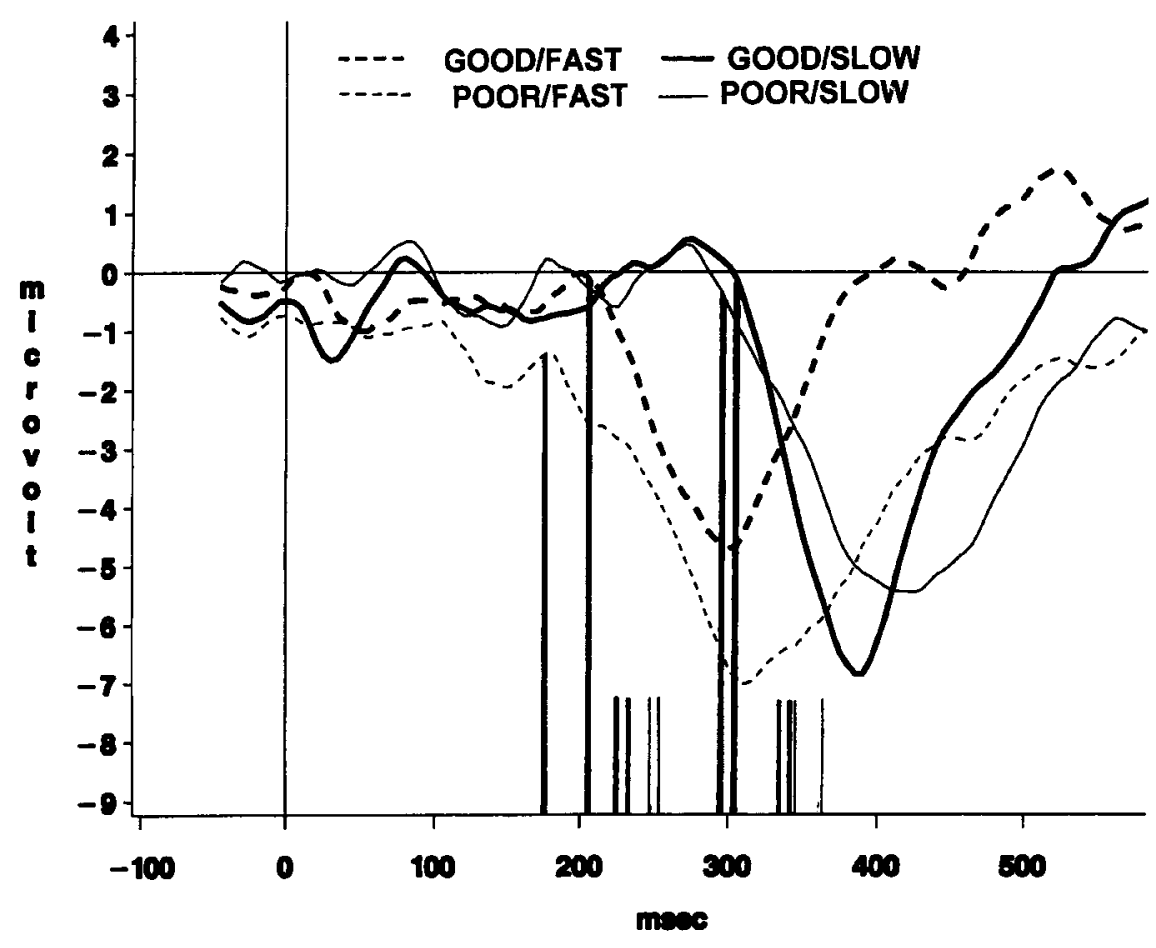

Figure 4. Grand average of the LRPs for different performance groups (good, subjects with $6 \%$ mean error rate; poor, subjects with $20 \%$ mean error rate) subdivided for slow (above median RT) and fast (below median RT) responses. The LRPs are pooled across modalities for a better overview. The two-segment character of all LRP curves between stimulus onset and LRP peak is obvious. The estimated onset values are marked with long vertical bars for the SR method and with short thick (BD) and thin (PP) vertical bars for the other methods. (Negativity = correct lateralization is plotted downward.) LRP peak and onset are delayed by about 100 msec on slow relative to fast responses. For slow responses, the LRP onset shows no group difference, whereas for fast responses the LRP begins slightly earlier for poor than for good subjects. This is revealed in the onsets estimated with the SR and (fainter) with the BD methods, but not with the PP method.

for the poor subjects), suggesting that poor subjects developed early response tendencies after auditory stimuli that led to premature responses. Hence the ANOVA effect expected from Figure 5 is a group $\times$ speed $\times$ modality interaction.

Table 1 shows the ANOVA results for the LRP onset with the three methods. In all three methods, the expected main effect of response speed (which amounts to about $100 \mathrm{msec}$ ) is highly significant, though the $F$ value is remarkably lower in the PP estimation. All other effects are of marginal significance, which is due to the small group size.

Only the SR estimator yielded the expected performance group $\times$ speed interaction as a tendency $(F=4.41, p=$ .07): For fast responses, the LRP began $31 \mathrm{msec}$ earlier in poor $(176 \mathrm{msec})$ than in good subjects $(207 \mathrm{msec})$. Both the SR and (fainter) the BD estimator yielded the expected triple interaction (performance group $x$ modality $X$ speed) as tendencies. The PP method failed to reveal these trends; in contrast, it yielded a tendency for a group main effect $(F=4.06, p=.08)$ : For poor subjects, the LRP onset was estimated $15 \mathrm{msec}$ later $(309 \mathrm{msec})$ than for good sub- jects $(294 \mathrm{msec})$. This was not revealed with the other two estimators. Figure 4 shows that this effect stemmed clearly from the LRP peak, which was in fact delayed for poor subjects, and not from the onset, which rather shows the opposite trend. Hence the PP method suggests (though insignificant) a misleading result.

\section{Discussion and Conclusion}

The known BD and PP methods use only restricted parts of the LRP trace for estimating the LRP onset latency. This makes the onset estimation vulnerable to differences across conditions in early (before LRP onset) and late (after LRP onset) parts of the LRP time course. In particular, early trends, which are in the same direction as the LRP slope reaching the peak lead to small onset latencies, whereas early trends in the opposite direction lead to large onset latencies if estimated with the BD method. Differences in LRP peak amplitudes and LRP slopes after the onset particularly influence the onset estimation by means of the PP method. Both difficulties can be overcome with the introduced segmented regression (SR) method, which 


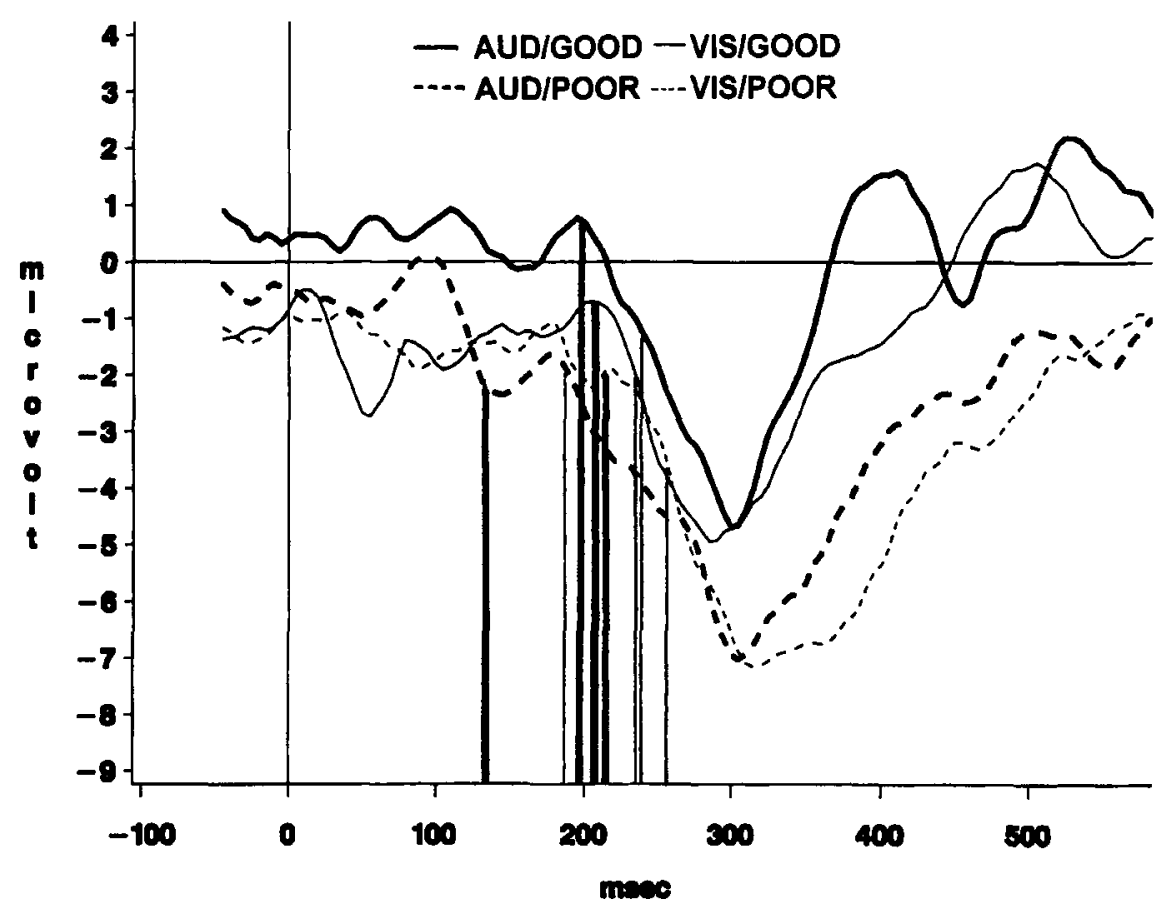

Figure 5. Grand average of the LRPs of good and poor subjects for the fast responses after presentation of visual and auditory stimuli. The LRP shows an earlier onset for poor than for good subjects only after presentation of auditory stimuli, as is also revealed in the SR (thick lines) and BD (thin lines) estimators.

estimates the LRP by a linear regression with two straight lines, where the onset is defined as the intersection of the two lines. A comparison of the SR method with the other two shows in addition, that the SR method is able to detect even subtle differences in LRP onset across conditions even with few subjects. The BD estimator was slightly less sensitive, while the PP method was rather insensitive to such differences. Rather, the latter estimator yielded misleading results, which were influenced by LRP peak latency effects. The fact that the $50 \%$ peak estimator is sensitive to the slope of the LRP was also considered by Smulders et al. (1996). They also stated that the method is invalid in case of true slope differences across conditions, as is illustrated in our example. Smulders et al. mentioned that other measures, like a " $12.5 \%$ LRP peak latency measure" (the point at which the LRP reaches $12.5 \%$ of the peak amplitude), which is less influenced by the peak latency, should be compared with the $50 \%$ measure before one draws conclusions about possible LRP onset effects between conditions. However, even a $12.5 \%$ measure cannot easily deal with the above mentioned influences of different LRP peak amplitudes across conditions. Altogether it is evident that particularly the PP method seems to be more sensitive to effects on LRP peak latency (and thereby to effects on RT), whereas the SR method is particularly sensitive to effects on the LRP onset latency. Since LRP onset seems to be sensitive to the central decision process (as argued in the introduction), the BD method seems the appropriate method to assess effects on central decision time rather than on RT.

The present method has been developed for very simple two-alternative choice tasks with only one stimulus attribute (name) being relevant. In some paradigms, however, several stimulus attributes may be task relevant. These attributes may be processed with different speeds, also providing different inputs to the response preparation system that are separated in time. In such conditions, more than two segments of the LRP may be visible, as, for example, in de Jong et al. (1988). Such conditions would consequently require a model with three (or even more) instead of two straight lines for regression. Hence the adequate model for regression (two, three, or even more lines) depends on the paradigm and the inspection of the grand mean wave shapes.

Another issue worth considering is whether straight lines are adequate for the regression, since the real data are usually composed of smooth curves. In further studies, we plan to test polynomial or other nonlinear regressions against the simple linear regressions used in the present paper.

A further issue to be explored is the robustness of the procedure in the presence of noise. Such noise can be seen in Figure 1, in the large baseline fluctuations, for example. 
Table 1

Analysis of Variance Results for the LRP Onset Latency, Estimated With the Three Different Methods

\begin{tabular}{|c|c|c|c|c|c|c|}
\hline \multirow[b]{3}{*}{ Effect } & \multicolumn{6}{|c|}{ Method } \\
\hline & \multicolumn{2}{|c|}{ SR } & \multicolumn{2}{|c|}{$\mathrm{BD}$} & \multicolumn{2}{|c|}{ PP } \\
\hline & $F$ & $p$ & $F$ & $p$ & $F$ & $p$ \\
\hline$P$ & 0.65 & .44 & 0.00 & 1.00 & 4.06 & .08 \\
\hline $\mathrm{M}$ & 3.66 & .09 & 5.49 & .05 & 0.05 & .84 \\
\hline $\mathrm{P} \times \mathrm{M}$ & 1.78 & .22 & 3.88 & .08 & 0.30 & .60 \\
\hline $\mathbf{S}$ & 136.97 & .00 & 191.82 & .00 & 53.65 & .00 \\
\hline $\mathbf{P} \times \mathbf{S}$ & 4.41 & .07 & 1.17 & .31 & 0.31 & .59 \\
\hline$M \times S$ & 0.13 & .73 & 1.99 & .20 & 0.10 & .76 \\
\hline $\mathbf{P} \times \mathbf{M} \times \mathbf{S}$ & 4.94 & .06 & 3.92 & .08 & 1.96 & .20 \\
\hline
\end{tabular}

Note-SR, segmented regression; BD, baseline deviation; PP, peak proportion. P, performance group (good, poor); M, modality (visual, auditory); S, speed (slow, fast). The factors expected to yield significant effects are given in boldface.

In particular, it seems to be important how many EEG epochs ("sweeps") are necessary to get a stable and reliable estimate of the model parameters. This is a particularly relevant question in error conditions. Here usually very small sweep numbers occur, since people usually commit only few errors per block. It also remains open for further research whether the lack of significant differences between conditions (e.g., in Table 1) is meaningful or merely due to a lack of power.

Finally, it is suggested that one combine the proposed SR method with the above mentioned jackknife method of Miller et al. (in press), since the jackknife method has the crucial advantage of estimating onsets in noisereduced wave shapes, whereas the SR method has the advantage of yielding a better absolute measure for onset values than the BD method (which is the basis for onset estimation in the jackknife method).

In summary, the SR method has the advantage that it estimates the LRP onset without being influenced by trends and amplitudes before and after the onset. In future applications, the SR method should be tested also with three-segment or nonlinear models in order to estimate the onset of early LRP trends relative to a zero (trendless) baseline. Finally, the combination of the SR and the jackknife methods should be explored further.

\section{REFERENCES}

Coles, M. G. H., \& Gratton, G. (1986). Cognitive psychophysiology and the study of states and processes. In G. R. J. Hockey, A. W. K. Gaillard, \& M. G. H. Coles (Eds.), Energetics and human information processing (pp. 409-424). Dordrecht: Martinus Nijhoff.

DE Jong, R., Wierda, M., Mulder, G., \& Mulder, L. J. M. (1988). Use of partial stimulus information in response processing. Journal of Experimental Psychology: Human Perception \& Performance, 14, 682-692.

EIMER, M. (1998). The lateralized readiness potential as an on-line measure of central response activation processes. Behavior Research Methods, Instruments, \& Computers, 30, 146-156.

Falkenstein, M., Hohnsbein, J., \& Hoormann, J. (1994). Effects of choice complexity on different subcomponents of the late positive complex of the event-related potential. Electroencephalography \& Clinical Neurophysiology, 92, 148-160.

Falkenstein, M., Hohnsbein, J., \& Hoormann, J. (1996). Reflections of performance quality in pre- and poststimulus ERP components. In C. Ogura, Y. Koga, \& M. Shimokochi (Eds.), Recent advances in event-related brain potential research (pp. 152-156). Amsterdam: Elsevier.

Hohnsbein, J., Falkenstein, M., Hoormann, J., \& Blanke, L. (1991). Effects of crossmodal divided attention on late ERP components: I. Simple and choice reaction tasks. Electroencephalography \& Clinical Neurophysiology, 78, 438-446.

Leuthold, H., \& SOMMER, W. (1993). Stimulus presentation rate dissociates sequential effects in event-related potentials and reaction times. Psychophysiology, 30, 510-517.

Meyer, D. E., Osman, A. M., Irwin, D. E., \& Yantis, S. (1988). Modern mental chronometry. Biological Psychology, 26, 3-67.

Miller, J., Patterson, T., \& Ulrich, R. (1998). A jackknife-based method for measuring LRP onset latency differences. Psychophysiology, 35, 99-115.

Osman, A. M., \& Moore, C. M. (1993). The locus of dual-task interference: Psychological refractory effects on movement-related brain potentials. Journal of Experimental Psychology: Human Perception \& Performance, 19, 1292-1312.

Seber, G. A. F., \& WILD, C. J. (1989). Nonlinear regression. New York: Wiley.

Smid, H. G. O. M., Mulder, G., \& Mulder, L. J. M. (1987). The continuous flow model revisited: Perceptual and central motor aspects. In R. Johnson, Jr., J. W. Rohrbaugh, \& R. Parasuraman (Eds.), Current trends in event-related potential research (EEG Suppl. 40, pp. 270278). Amsterdam: Elsevier.

Smulders, F. T. Y., Kenemans, J. L., \& KoK, A. (1996). Effects of task variables on measures of the mean onset latency of LRP depend on the scoring method. Psychophysiology, 33, 194-205.

Ulrich, R., Moore, C., \& Osman, A. (1993). Lateralisiertes Bereitschaftspotential und Reaktionsgeschwindigkeit bei partieller Vorinformation eines Handlungsparameters. Zeitschrift für experimentelle und angewandte Psychologie, 40, 310-325. 